\title{
Coherent elastic excitation of spin waves
}

\author{
Akashdeep Kamra, ${ }^{1}$ Hedyeh Keshtgar, ${ }^{2}$ Peng Yan, ${ }^{1}$ and Gerrit E. W. Bauer ${ }^{3,1}$ \\ ${ }^{1}$ Kavli Institute of NanoScience, Delft University of Technology, Lorentzweg 1, 2628 CJ Delft, The Netherlands \\ ${ }^{2}$ Institute for Advanced Studies in Basic Science, 45195 Zanjan, Iran \\ ${ }^{3}$ Institute for Materials Research and WPI-AIMR, Tohoku University, Sendai 980-8577, Japan \\ (Received 18 December 2014; revised manuscript received 6 February 2015; published 13 March 2015)
}

\begin{abstract}
We model the injection of elastic waves into a ferromagnetic film $(\mathrm{F})$ by a nonmagnetic transducer $(\mathrm{N})$. We compare the configurations in which the magnetization is normal and parallel to the wave propagation. The lack of axial symmetry in the former results in the emergence of evanescent interface states. We compute the energy-flux transmission across the $\mathrm{N} \mid \mathrm{F}$ interface and sound-induced magnetization dynamics in the ferromagnet. We predict efficient acoustically induced pumping of spin current into a metal contact attached to F.
\end{abstract}

DOI: 10.1103/PhysRevB.91.104409

PACS number(s): 75.80.+q, 75.70.Cn, 75.30.Ds

\section{INTRODUCTION}

The macroscopic magnetic moment of a ferromagnet results from a symmetry-broken ground state in which the constituent spins align by the exchange interaction [1]. The underlying crystal lattice breaks the rotational invariance of the magnetic order. Owing to spin-orbit interaction and dipolar fields, the spins experience elastic deformations in the form of a magnetoelastic coupling (MEC). Vice versa, the lattice is affected by the magnetization in the form of, e.g., magnetostriction. The MEC appears to be the dominant cause for Gilbert damping [2] of the magnetization dynamics of insulators and plays the key role in equilibration of the magnetic system with its surroundings [3]. It also offers elastic control of magnetization dynamics.

While the coupled elastic and magnetic dynamics was first investigated half a century ago $[3,4]$, interest in this area has been rekindled by improved material growth and fabrication methods. Uchida et al. [5] induced spin pumping by longitudinal acoustic waves injected into a ferromagnetic insulator, suggesting MEC to be a possible mechanism behind the transverse spin Seebeck effect [6]. Weiler et al. excited ferromagnetic resonance (FMR) in a cobalt film by pulsed surface acoustic waves [7]. Static strains induce effective magnetic fields that can be used to manipulate the magnetization [8]. Full magnetization reversal of a magnetic film on a cantilever by magnetomechanical coupling has been predicted [9].

While several authors [3,4] investigated magnetoelastic waves (MEWs) in magnetic bulk crystals, boundary conditions and finite size effects, which are essential to understand ultrathin films and nanostructures, have seldom been addressed [10]. We previously proposed [11] a scattering theory for MEW propagation analogous to the Landauer-Büttiker formalism for electronic transport in mesoscopic systems [12].

Here we study the excitation and propagation of MEWs in a ferromagnet by a nonmagnetic transducer that injects elastic waves into the ferromagnet. MEWs are generated at the interface by MEC-induced hybridization between the spin and elastic waves. The mixing is resonantly enhanced around the (anti)crossing of the spin and lattice wave dispersion relations at which fully mixed magnon polarons (MPs) are generated. Far from this region, the MEWs can be considered dominantly magnonic (spin) or phononic (elastic). Because of their mixed character, MPs can be excited by exposing the ferromagnet to sound waves.

The equations of motion for MEWs propagating in arbitrary directions are derived in Sec. II A. Two special cases of interest are waves traveling perpendicular to (configuration 1) and along (configuration 2) the equilibrium magnetization, since they can be solved analytically and offer direct physical insights. Here we focus on configuration 1 and compare results with configuration 2 where appropriate [11]. Physically, configuration 1 differs from configuration 2 by the broken axial symmetry that causes a mixing of the right and left precessing spin waves. We formulate the basis for a scattering matrix theory in Sec. II B and derive magnetoelastic boundary conditions (BCs) in Sec. II C. The energy transport across a nonmagnet|ferromagnet interface and the resulting excitation of MEWs are given in Sec. III A. Considering thin film ferromagnets, we investigate finite size effects such as standing wave excitations in Sec. III B. We conclude with a discussion in Sec. IV.

\section{THEORETICAL METHOD}

\section{A. Magnetoelastic waves in ferromagnets}

In this section, we recapitulate the continuum theory of low energy excitations in a ferromagnet including the magnetoelastic coupling. We closely follow Kittel [4] to obtain the coupled equations of motion for magnetization (M) and displacement (R) fields. An applied magnetic field and easy-axis anisotropy, and thus the equilibrium magnetization direction, is chosen along the $\hat{\mathbf{z}}$ direction (see Fig. 1).

\section{Energy density in a ferromagnet}

The free energy density $\mathcal{H}$ has contributions from the Zeeman interaction, magnetic anisotropy, exchange interaction, MEC, and elastic energy,

$$
\mathcal{H}=\mathcal{H}_{\mathrm{Z}}+\mathcal{H}_{\mathrm{an}}+\mathcal{H}_{\mathrm{ex}}+\mathcal{H}_{\mathrm{MEC}}+\mathcal{H}_{\mathrm{el}} \text {. }
$$

For small deviations from equilibrium $\left(M_{x, y} \ll M_{z} \approx M_{s}\right.$, the saturation magnetization), Zeeman plus anisotropy energy densities read [4]

$$
\mathcal{H}_{\mathrm{Z}}+\mathcal{H}_{\mathrm{an}}=\frac{\omega_{0}}{2 \gamma M_{s}}\left(M_{x}^{2}+M_{y}^{2}\right)
$$




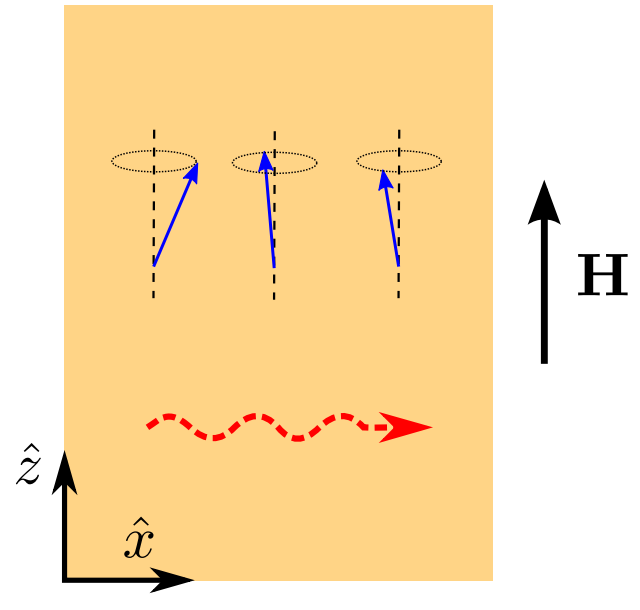

(a) Config. 1

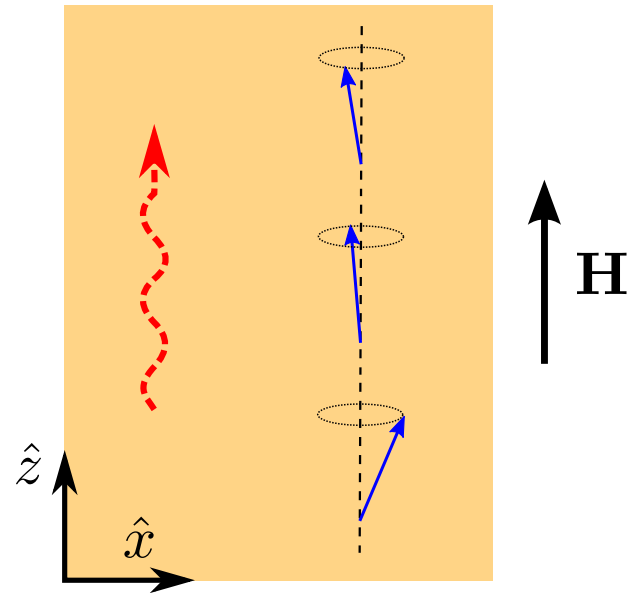

(b) Config. 2

FIG. 1. (Color online) Two canonical configurations for magnetoelastic wave propagation in ferromagnets. The magnetization is saturated along the $z$ axis by a magnetic field $\mathbf{H}$. The blue arrows depict an instantaneous magnetization texture with spin wave excitations. The red wavy arrow represents wave propagation along (a) $\hat{\mathbf{x}}$ and (b) $\hat{\mathbf{z}}$.

where $\omega_{0}=\gamma \mu_{0} H$ is the ferromagnetic resonance frequency, $H$ is the magnitude of the external plus the anisotropy fields along $\hat{\mathbf{z}}, \mu_{0}$ is the vacuum permeability, and $\gamma(>0)$ is the gyromagnetic ratio. The exchange energy density can be expressed as [13]

$$
\mathcal{H}_{\mathrm{ex}}=\frac{A}{M_{s}^{2}}\left[\left(\nabla M_{x}\right)^{2}+\left(\nabla M_{y}\right)^{2}\right],
$$

in terms of the exchange constant $A$. The elastic energy density for an isotropic solid reads

$$
\mathcal{H}_{\mathrm{el}}=\frac{1}{2} \rho_{F}(\dot{\mathbf{R}} \cdot \dot{\mathbf{R}})^{2}+\frac{\lambda_{F}}{2}\left(\sum_{i} S_{i i}\right)^{2}+\mu_{F} \sum_{i j} S_{i j}^{2},
$$

in terms of the density $\rho_{F}$, the Lame's constants $\lambda_{F}$ and $\mu_{F}$, and the components of the strain tensor [14] $S_{i j}=1 / 2\left(\partial R_{i} / \partial x_{j}+\right.$ $\left.\partial R_{j} / \partial x_{i}\right)$.

For cubic symmetry the MEC energy density is parametrized by the MEC constants $b_{1,2}$ as

$$
\begin{aligned}
\mathcal{H}_{\mathrm{MEC}}= & \frac{b_{1}}{M_{s}^{2}} \sum_{i} M_{i}^{2} S_{i i}+\frac{b_{2}}{M_{s}^{2}} \sum_{i \neq j} M_{i} M_{j} S_{i j} \\
& +\frac{r_{0}}{3 M_{s}^{2}} \frac{\partial A}{\partial r}\left[\left(\nabla M_{x}\right)^{2}+\left(\nabla M_{y}\right)^{2}\right]\left(\sum_{i} S_{i i}\right), \\
\approx & \frac{2 b_{2}}{M_{s}}\left(M_{x} S_{x z}+M_{y} S_{y z}\right),
\end{aligned}
$$

where $r$ is the distance between nearest neighbor spins with equilibrium value $r_{0}$, and only terms linear in $M_{x, y}$ have been retained in the second step. The effects of the nonlinear terms have been considered elsewhere [15]. The (disregarded) last term in Eq. (5) represents the MEC [16] mediated by the dependence of the exchange integral on $r$. Considering the linear terms only, we may interpret the MEC as an effective Zeeman field with its $x$ and $y$ components proportional to $S_{x z}$ and $S_{y z}$, respectively.

\section{Equations of motion}

The Hamilton equations of motion for the energy density defined above read [4,17]

$$
\begin{gathered}
\dot{M}_{x}=\omega_{0} M_{y}-D \nabla^{2} M_{y}+b_{2} \gamma\left(\frac{\partial R_{y}}{\partial z}+\frac{\partial R_{z}}{\partial y}\right), \\
\dot{M}_{y}=-\omega_{0} M_{x}+D \nabla^{2} M_{x}-b_{2} \gamma\left(\frac{\partial R_{x}}{\partial z}+\frac{\partial R_{z}}{\partial x}\right), \\
\rho_{F} \ddot{R}_{x}=\mu_{F} \nabla^{2} R_{x}+\left(\lambda_{F}+\mu_{F}\right) \frac{\partial}{\partial x} \nabla \cdot \mathbf{R}+\frac{b_{2}}{M_{s}} \frac{\partial M_{x}}{\partial z}, \\
\rho_{F} \ddot{R}_{y}=\mu_{F} \nabla^{2} R_{y}+\left(\lambda_{F}+\mu_{F}\right) \frac{\partial}{\partial y} \nabla \cdot \mathbf{R}+\frac{b_{2}}{M_{s}} \frac{\partial M_{y}}{\partial z}, \\
\rho_{F} \ddot{R}_{z}=\mu_{F} \nabla^{2} R_{z}+\left(\lambda_{F}+\mu_{F}\right) \frac{\partial}{\partial z} \nabla \cdot \mathbf{R} \\
+\frac{b_{2}}{M_{s}}\left(\frac{\partial M_{x}}{\partial x}+\frac{\partial M_{y}}{\partial y}\right),
\end{gathered}
$$

where $D=2 A \gamma / M_{s}$ is the spin wave stiffness. We disregard dissipation since we are primarily interested in magnetic insulators such as yttrium iron garnet (YIG) with very weak Gilbert and mechanical damping. The equations above demonstrate coupling between all five field variables that renders an analytic solution intractable. In the following we therefore focus on two configurations corresponding to wave propagation orthogonal to and along the equilibrium magnetization direction $(z)$ as shown in Fig. 1.

Configuration 1. For wave propagation along the $x$ direction the partial derivatives with respect to $y$ and $z$ in Eqs. (6)-(10) vanish and only the transverse displacement $R_{z}$ couples to the magnetization dynamics. The MEC [Eq. (5)] reduces to

$$
\mathcal{H}_{\mathrm{MEC}}=\frac{b_{2}}{M_{s}} M_{x} \frac{\partial R_{z}}{\partial x}
$$


which is not invariant under rotation about the $z$ direction. With constant coefficients the equations of motion are solved by plane waves $B(x, t)=\operatorname{Re}\left[b(k, \omega) e^{i(k x-\omega t)}\right]$ and can be written as a matrix equation $\mathcal{A} \chi=0$,

$$
\left(\begin{array}{ccc}
i \omega & \omega_{m} & 0 \\
-\omega_{m} & i \omega & -i b_{2} \gamma k \\
i b_{2} k /\left(\rho_{F} M_{s}\right) & 0 & \omega^{2}-\omega_{p}^{2}
\end{array}\right)\left(\begin{array}{c}
m_{x} \\
m_{y} \\
r_{z}
\end{array}\right)=\left(\begin{array}{l}
0 \\
0 \\
0
\end{array}\right),
$$

where $\quad \omega_{m}=\omega_{m}(k)=\omega_{0}+D k^{2} \quad$ and $\quad \omega_{p}=\omega_{p}(k)=$ $k \sqrt{\mu_{F} / \rho_{F}}$ are the uncoupled magnonic and phononic dispersion relations.

Configuration 2. For waves propagating along the $z$ direction both transverse components $R_{x}$ and $R_{y}$ couple to the magnetization dynamics. The MEC [Eq. (5)] then reduces to the axially symmetric form

$$
\mathcal{H}_{\text {MEC }}=\frac{b_{2}}{M_{s}}\left(M_{x} \frac{\partial R_{x}}{\partial z}+M_{y} \frac{\partial R_{y}}{\partial z}\right) .
$$

By the transformation $M^{ \pm}=M_{x} \pm i M_{y}$ and $R^{ \pm}=R_{x} \pm i R_{y}$, the $4 \times 4$ matrix equation is block-diagonalized into two $2 \times 2$ equations [11],

$$
\left(\begin{array}{cc}
i\left(\omega-\sigma \omega_{m}\right) & \sigma \gamma b_{2} k \\
i b_{2} k / \rho_{F} M_{s} & \omega^{2}-\omega_{p}^{2}
\end{array}\right)\left(\begin{array}{c}
m^{\sigma} \\
r^{\sigma}
\end{array}\right)=0,
$$

where $\sigma= \pm$ is a chirality index. $m^{+}$denotes the spin waves that precess "with" the magnetic field, while $m^{-}$represents counter-rotating modes with frequency $\omega=-\omega_{m}(k)$. Since $k$ is imaginary for any (positive) frequency, these waves are always evanescent and cannot exist in the bulk of the ferromagnet. $r^{+}$represents the right and $r^{-}$the left circularly polarized elastic waves.

Comparison between configurations 1 and 2. Since we consider waves along symmetry directions, only the elastic shear waves couple to the magnetization in both cases [18]. The three eigenmodes for configuration 1 , as will be discussed in Sec. II C, correspond to the three coupled variables [see Eq. (12)], and two eigenmodes for configuration 2 [see Eq. (14)]. The right and left precessing magnetoelastic modes are uncoupled under the axial symmetry of configuration 2 , but they become mixed when this symmetry is broken in configuration 1 . The elastic displacement $r_{z}$ then couples to the evanescent $m^{-}$as well as the propagating $m^{+}$waves. This mixing is important in the "ultrastrong" coupling regime in which the rotating wave approximation, i.e., the neglect of the \pm coupling, breaks down. Typically, the FMR frequency is much higher than the frequency equivalent of MEC strength, and the rotating wave approximation is valid. Nevertheless, the evanescent waves are necessary to formulate proper boundary conditions and affect the conversion of acoustic to magnetic energy at the interfaces.

\section{Magnetoelastic eigenmodes}

Diagonalization of Eq. (12) leads to the dispersion relations of the magnetoelastic waves (MEWs),

$$
\omega_{ \pm}=\sqrt{\frac{\omega_{m}^{2}+\omega_{p}^{2}}{2} \pm \sqrt{\left(\frac{\omega_{m}^{2}-\omega_{p}^{2}}{2}\right)^{2}+\frac{b_{2}^{2} k^{2} \gamma \omega_{m}}{\rho_{F} M_{s}}} .}
$$

The wave vectors $k_{a}$ of the eigenmodes at frequency $\omega$, where the subscript $a$ labels the eigenmodes, are obtained by inverting the dispersion relation [Eq. (15)], as will be discussed in Sec. II C. The corresponding eigenvectors $\chi_{a}$ are

$$
\chi_{a}=\left(\begin{array}{c}
m_{x} \\
m_{y} \\
r_{z}
\end{array}\right)=N_{a}\left(\begin{array}{c}
i b_{2} \gamma k_{a} \omega_{m a} /\left(\omega^{2}-\omega_{m a}^{2}\right) \\
b_{2} \gamma k_{a} \omega /\left(\omega^{2}-\omega_{m a}^{2}\right) \\
1
\end{array}\right),
$$

where $N_{a}$ is a dimensionless normalization factor, $\omega_{m a} \equiv$ $\omega_{m}\left(k_{a}\right)$, and the eigenmodes consist of elliptical magnetization precession around $z$ coupled with the elastic shear mode along $z$. The dispersion [Eq. (15)] is plotted in Fig. 2 for parameters appropriate for YIG: $M_{s}=1.4 \times$ $10^{5} \mathrm{~A} / \mathrm{m}, b_{2}=5.5 \times 10^{5} \mathrm{~J} / \mathrm{m}^{3}, D=8.2 \times 10^{-6} \mathrm{~m}^{2} / \mathrm{s}, H=$ $8 \times 10^{4} \mathrm{~A} / \mathrm{m}, \gamma=2.8 \times 10^{10} \mathrm{~Hz} / \mathrm{T}, \rho_{F}=5170 \mathrm{~kg} / \mathrm{m}^{3}$, and $\mu_{F}=74$ GPa [19-21]. When the MEC is weak, the branches in the dispersion diagram are quite close to the uncoupled dispersion relations $\omega_{m}$ or $\omega_{p}$ [see Eq. (15)] in much of the phase space [the $\mathrm{W}$ regions in Fig. 2(b)]. Here the mode with frequencies close to $\omega_{m}\left(\omega_{p}\right)$ is dominantly magnonic (phononic). In the crossing regime, i.e., when $4 b_{2}^{2} k^{2} \gamma \omega_{m} / \rho_{F} M_{s} \gtrsim\left(\omega_{m}^{2}-\omega_{p}^{2}\right)^{2}$, the excitations hybridize [the $\mathrm{S}$ region in Fig. 2(b)]. We refer to the quasiparticle close to $k_{0}$ at which the uncoupled dispersions cross as "magnon polaron (MP)". Since the uncoupled magnon dispersion is very flat compared to that of the phonons, we may define a narrow $M$ region in $\omega$ space [Fig. 2(b)] in which the magnon character dominates both excitation modes, while the phonon character is suppressed, leading to a pseudoband gap for quasiphononic excitations [see also Fig. 4(b)]. For spin wave stiffness $D \ll \omega_{0} / k_{0}^{2}, k_{0} \approx \omega_{0} \sqrt{\rho_{F} / \mu_{F}}$, and the $\mathrm{M}$ region covers the frequency interval $\left|\omega-\omega_{0}\right| \lesssim \sqrt{b_{2}^{2} \omega_{0} \gamma / 4 \mu_{F} M_{s}} \approx 24 \mathrm{MHz}$.

\section{B. Energy flux and eigenmode normalization}

Energy conservation can be expressed by the continuity equation [3]

$$
\frac{\partial \mathcal{H}}{\partial t}+\nabla \cdot \mathbf{F}=0
$$

where the energy flux $\mathbf{F}=F_{x} \hat{\mathbf{x}}$,

$$
\begin{aligned}
F_{x}= & -\left[\frac{2 A}{M_{s}^{2}}\left(\frac{\partial M_{x}}{\partial t} \frac{\partial M_{x}}{\partial x}+\frac{\partial M_{y}}{\partial t} \frac{\partial M_{y}}{\partial x}\right)\right. \\
& \left.+\frac{\partial R_{z}}{\partial t}\left(\mu_{F} \frac{\partial R_{z}}{\partial x}+b_{2} \frac{M_{x}}{M_{s}}\right)\right] .
\end{aligned}
$$

For real $k, B(x, t)=\left[b(k, \omega) e^{i(k x-\omega t)}+b^{*}(k, \omega) e^{-i(k x-\omega t)}\right] / 2$, whence the time-averaged energy flux $\overline{\mathbf{F}}$ is constant,

$\bar{F}_{x}=\frac{A \omega k}{M_{s}^{2}}\left(\left|m_{x}\right|^{2}+\left|m_{y}\right|^{2}\right)+\frac{\mu_{F} \omega k}{2}\left|r_{z}\right|^{2}+\frac{b_{2} \omega}{2 M_{s}} \operatorname{Im}\left(r_{z}^{*} m_{x}\right)$.

For the eigenmode $\chi_{a}$,

$$
\begin{aligned}
\bar{F}_{x}^{a}= & N_{a}^{2}\left[\frac{\mu_{F} k_{a} \omega}{2}+\frac{A b_{2}^{2} \gamma^{2}}{M_{s}^{2}} \frac{k_{a}^{3} \omega\left(\omega^{2}+\omega_{m a}^{2}\right)}{\left(\omega^{2}-\omega_{m a}^{2}\right)^{2}}\right. \\
& \left.+\frac{b_{2}^{2} \gamma}{2 M_{s}} \frac{k_{a} \omega \omega_{m a}}{\omega^{2}-\omega_{m a}^{2}}\right] .
\end{aligned}
$$




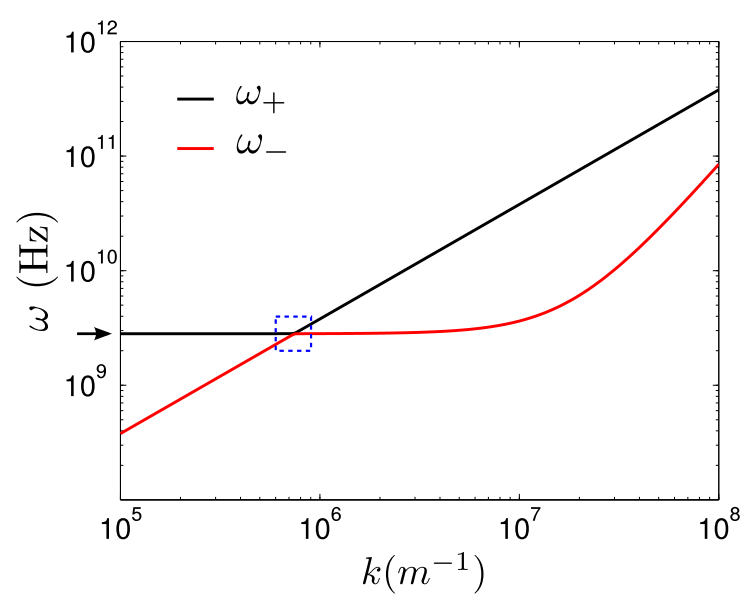

(a)

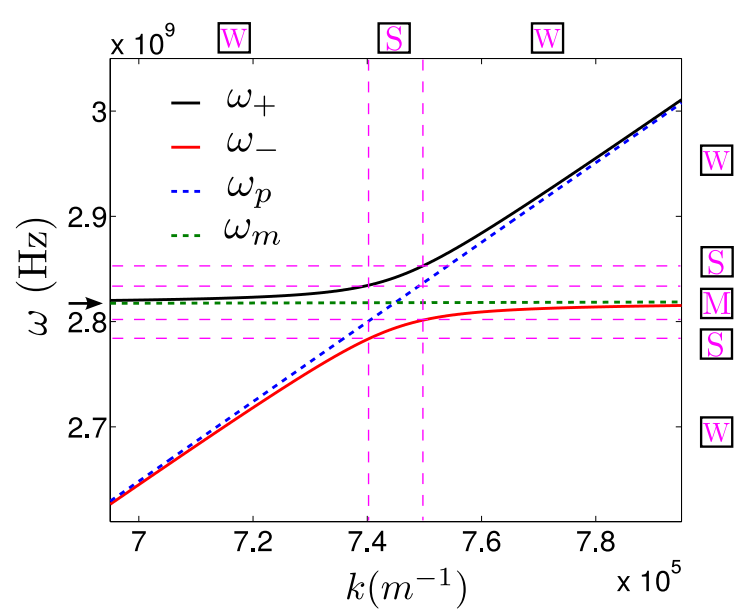

(b)

FIG. 2. (Color online) (a) Dispersion relation for magnetoelastic waves (MEWs) in a ferromagnet calculated using Eq. (15) and parameters for YIG. The arrow on the ordinate indicates the FMR frequency $\omega_{0}$. The blue dashed box is expanded in (b) to reveal the anticrossing. The dashed lines denote the unperturbed magnonic $\left(\omega_{m}\right.$, green line $)$ and phononic $\left(\omega_{p}\right.$, blue line) dispersion relations, while the solid lines represent the coupled MEWs. The $\omega-k$ space can be classified into three regions: (i) the W region, where the MEWs can be considered quasiphononic or quasimagnonic, (ii) the S region, where the MEWs have a mixed character, and (iii) the $\mathrm{M}$ region, where both excitations are quasimagnonic, leading to a pseudoband gap for quasiphononic excitations.

$\bar{F}$ vanishes for imaginary $k$, i.e., evanescent waves that store, but do not propagate, energy. Equations (19) and (20) reduce to the flux carried by purely elastic (spin) waves in the limit $b_{2} \rightarrow 0$ and $\omega \rightarrow \omega_{p}\left(\omega_{m}\right)$. In transport theory it is convenient to choose the normalization factors $N_{a}$ such that each eigenmode carries unit energy flux, i.e., $\bar{F}_{x}^{a}=1 \mathrm{~W} / \mathrm{m}^{2}$ [Eq. (20)]. When interested in the amplitude or the energy density, choosing a normalization factor of $N_{a}=1$ may be simpler. The calculated physical quantities are of course independent of the normalization chosen. In the following we will employ flux normalized representation for the propagating waves.

\section{Boundary conditions and acoustic actuation of MEWs}

So far we have discussed MEWs in the bulk of a ferromagnet. Next, we derive the interface connection rules for a nonmagnetic transducer $(\mathrm{N})$ attached to a ferromagnet $(\mathrm{F})$. The required boundary conditions (BCs) can be obtained by integrating the equations of motion over the abrupt interface with discontinuous constitutive parameters. This is equivalent to demanding continuity of the energy flux [Eq. (18)] across the interface [3]. The first BC corresponds to zero spin wave angular momentum flux at the interface or "free" BC for the magnetization,

$$
\left.\frac{\partial M_{x, y}}{\partial x}\right|_{F}=0 .
$$

Here, we disregard the anisotropies that could "pin" the magnetization at the interface. Continuity of mass velocity (or, equivalently, displacement) at the interface implies

$$
\left.\frac{\partial R_{z}}{\partial t}\right|_{F}=\left.\frac{\partial R_{z}}{\partial t}\right|_{N} .
$$

The third BC is the continuity of stress at the interface,

$$
\left.\left(\mu_{F} \frac{\partial R_{z}}{\partial x}+b_{2} \frac{M_{x}}{M_{s}}\right)\right|_{F}=\left.\mu_{N} \frac{\partial R_{z}}{\partial x}\right|_{N} .
$$

These BCs should be satisfied for all frequencies. The wave numbers $k_{a}$ in $\mathrm{F}$ corresponding to a given frequency $\omega(>0)$ of the elastic wave incident from $\mathrm{N}$ are obtained by inverting the MEW dispersion relation [Eq. (15)]. The secular equation

$$
\begin{aligned}
0= & \frac{\mu_{F} D^{2}}{\rho_{F}} k^{6}+\left[\frac{2 \omega_{0} D \mu_{F}}{\rho_{F}}-\frac{b_{2}^{2} \gamma D}{\rho_{F} M_{s}}-D^{2} \omega^{2}\right] k^{4} \\
& -\left[2 \omega^{2} \omega_{0} D+\frac{\mu_{F}}{\rho_{F}}\left(\omega^{2}-\omega_{0}^{2}\right)+\frac{b_{2}^{2} \gamma \omega_{0}}{\rho_{F} M_{s}}\right] k^{2} \\
& +\omega^{2}\left(\omega^{2}-\omega_{0}^{2}\right)
\end{aligned}
$$

is cubic in $k^{2}$, implying three (doubly degenerate) solutions. One of these solutions $\left(k_{1}\right)$ is real for all $\omega$, representing a propagating wave. It corresponds to the $\omega_{-}$branch of the dispersion [Fig. 2(a)] with limiting values $k_{1} \rightarrow \omega \sqrt{\rho_{F} / \mu_{F}}$ for $\omega<\omega_{0}$ and $k_{1} \rightarrow \sqrt{\left(\omega-\omega_{0}\right) / D}$ for $\omega>\omega_{0}$ for $b_{2} \rightarrow 0$. The second root $k_{2}$ corresponds to the upper $\omega_{+}$branch of the dispersion with, for $b_{2} \rightarrow 0$, limiting values $k_{2} \rightarrow$ $\sqrt{\left(\omega-\omega_{0}\right) / D}$ for $\omega \lesssim \omega_{0}$ and $k_{2} \rightarrow \omega \sqrt{\rho_{F} / \mu_{F}}$ for $\omega>\omega_{0}$, therefore evanescent for $\omega$ below and propagating above $\omega_{0}$. The third solution $k_{3} \rightarrow i \sqrt{\left(\omega+\omega_{0}\right) / D}$ in the limit $b_{2} \rightarrow 0$ is always evanescent and thus does not appear in the dispersion diagram [Fig. 2(a)].

\section{RESULTS}

\section{A. Acoustic energy transfer across $\mathbf{N} \mid \mathrm{F}$ interfaces}

Here we consider a ferromagnet $(\mathrm{F})$ in contact with a nonmagnetic transducer $(\mathrm{N})$ that injects elastic waves propagating along $\hat{\mathbf{x}}$ (see Fig. 3). Both $\mathrm{F}$ and $\mathrm{N}$ are semi-infinite (or with 


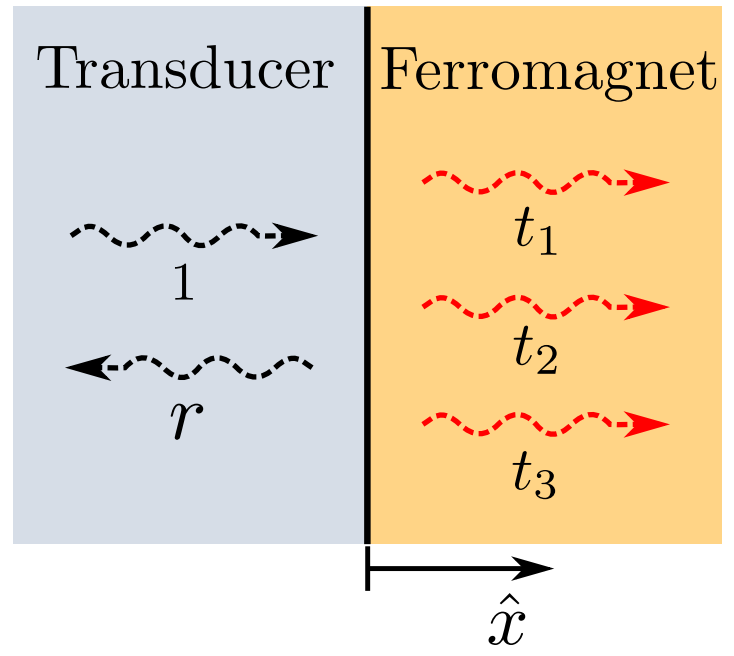

FIG. 3. (Color online) Scattering process at an N|F interface with magnetization along $\hat{\mathbf{z}}$. Linear polarized (along $\hat{\mathbf{z}}$ ) transverse acoustic waves generated in a nonmagnetic transducer $(\mathrm{N})$ impinge on a ferromagnet $(\mathrm{F})$ in the $x$ direction. The incident wave is partially reflected (without mode conversion) and partially transmitted into the $\mathrm{F}$ as three MEWs (shown as red wavy arrows). One of these MEWs is always propagating, the second one is evanescent or traveling depending on the frequency of the incident wave, while the third one is always evanescent.

a perfect absorber attached to the $\mathrm{F}$ side) so that only the $\mathrm{N} \mid \mathrm{F}$ interface at $x=0$ matters while there are no incoming propagating waves from $\mathrm{F}$. With these boundary conditions a flux-normalized sound wave in $\mathrm{N}$ (with parameters denoted by subscript $N$ ) reads

$$
\begin{aligned}
\psi_{N}(x \leqslant 0)= & \left(M_{x}, M_{y}, R_{z}\right)^{\top}=\sqrt{\frac{2}{\mu_{N} \omega k_{i}}}(0,0,1)^{\top} e^{i\left(k_{i} x-\omega t\right)} \\
& +r(\omega) \sqrt{\frac{2}{\mu_{N} \omega k_{i}}}(0,0,1)^{\top} e^{-i\left(k_{i} x+\omega t\right)},
\end{aligned}
$$

where $k_{i}=\omega \sqrt{\rho_{N} / \mu_{N}}$ is the wave number of the incident (and reflected) wave and $r(\omega)$ is the reflection coefficient calculated below. In $\mathrm{F}$ we have to consider the three MEWs derived above:

$$
\psi_{F}(x \geqslant 0)=\sum_{l=1,2,3} t_{l}(\omega) \chi_{l} e^{i\left(k_{l} x-\omega t\right)} .
$$

The propagating waves in Eq. (26) are assumed to be flux-normalized such that the reflection and transmission probabilities of the propagating waves are simply given by $|r|^{2}$ and $\left|t_{j}\right|^{2}$, leading to

$$
|r|^{2}+\sum_{j}\left|t_{j}\right|^{2}=1
$$

where the index $j$ runs over propagating modes only. The normalization factor $N_{a}$ has been chosen to be 1 for evanescent modes.

Imposing the four boundary conditions [Eqs. (21)-(23)] yields four equations for the four variables $r, t_{1,2,3}$,

$$
\sum_{l} t_{l} k_{l} \chi_{l}[1]=0,
$$

$$
\begin{gathered}
\sum_{l} t_{l} k_{l} \chi_{l}[2]=0, \\
\sum_{l} t_{l} \chi_{l}[3]=\sqrt{\frac{2}{\mu_{N} \omega k_{i}}}(1+r), \\
\sum_{l}\left(i \mu_{F} k_{l} t_{l} \chi_{l}[3]+\frac{b_{2}}{M_{s}} t_{l} \chi_{l}[1]\right)=i \mu_{N} k_{i} \sqrt{\frac{2}{\mu_{N} \omega k_{i}}}(1-r),
\end{gathered}
$$

with $\chi_{l}[m]$ denoting the $m$ th element of the vector $\chi_{l}$. The analytic solutions are unwieldy and not presented here. In Fig. 4(a) we plot the energy flux carried by the propagating waves for a junction of magnetic YIG and nonmagnetic gadolinium gallium garnet (GGG) with parameters $\rho_{N}=7085 \mathrm{~kg} / \mathrm{m}^{3}$ and $\mu_{N}=90 \mathrm{GPa}$ [22]. The small but finite acoustic mismatch causes partial reflection even far from the resonance without actuating the magnetization.

Figure 4(a) is very similar to the analogous plot for the symmetric configuration (configuration 2) considered in Ref. [11] in which circularly polarized MEWs propagate along the equilibrium magnetization direction. Far from the anticrossing, transmission is efficient into the quasiphononic excitation. The modes gradually change their character when approaching the anticrossing. Hence transmission into one branch increases at the cost of the other one. The evanescent modes apparently do not affect the steady state transmission even close to the anticrossing. We expect them to play a significant role in the transmitted energy current only in the ultrastrong coupling regime in which the MEC is of the order of $\omega_{0}$. However, this does not imply that the evanescent states may be neglected. They do store significant energy and should show up in the transients when actuation is carried out by ultrashort pulses. Furthermore, the presence of defects would mix the evanescent interface states with propagating ones. The MEWs are efficiently excited in the full frequency range [see Fig. 4(a)] including MPs, which are formed at about 2.79 and $2.84 \mathrm{GHz}$ [see Fig. 4(b)], although in contrast to the energy density in Fig. 4(b), the energy flux is still dominated by the lattice degree of freedom [see Fig. 4(c)].

\section{B. Excitation of spin waves in ferromagnetic films}

We now consider finite size effects in the device depicted in Fig. 5 in which F is bounded by the actuator on one side and air or vacuum on the other. Since we disregard damping, net energy transport through any cross section vanishes. $\mathrm{N}$ is still described by Eq. (25) while in F,

$$
\begin{aligned}
\psi_{F}(0 \leqslant x \leqslant d)= & \sum_{l=1,2,3}\left[t_{l}^{+} \chi_{l}\left(k_{l}\right) e^{i\left(k_{l} x-\omega t\right)}\right. \\
& \left.+t_{l}^{-} \chi_{l}\left(-k_{l}\right) e^{-i\left[k_{l}(x-d)+\omega t\right]}\right]
\end{aligned}
$$

with $k_{l}>0$ for traveling and $\operatorname{Im}\left(k_{l}\right)>0$ for evanescent waves. Since $\chi_{l}[1,2]\left(-k_{l}\right)=-\chi_{l}[1,2]$ and $\chi_{l}[3]\left(-k_{l}\right)=\chi_{l}[3]$, 


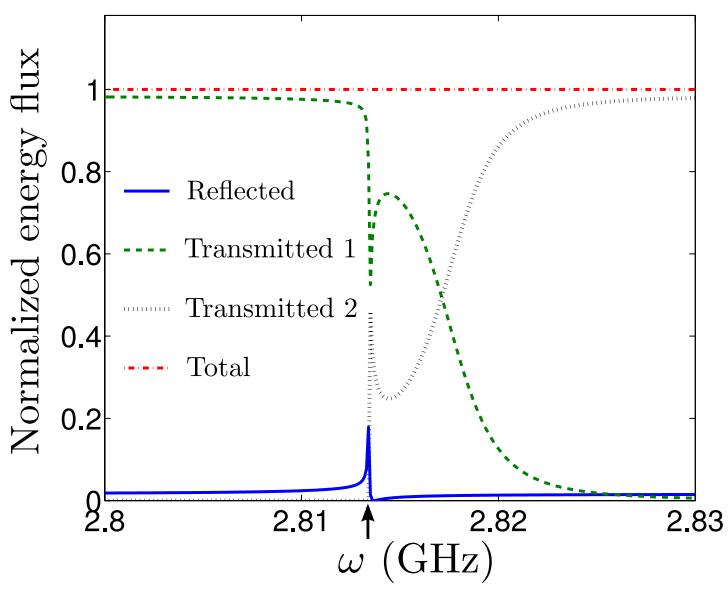

(a)

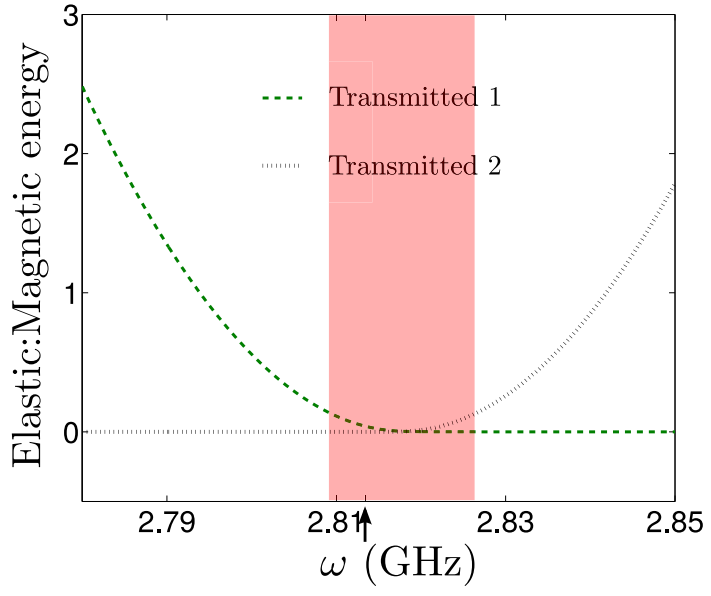

(b)

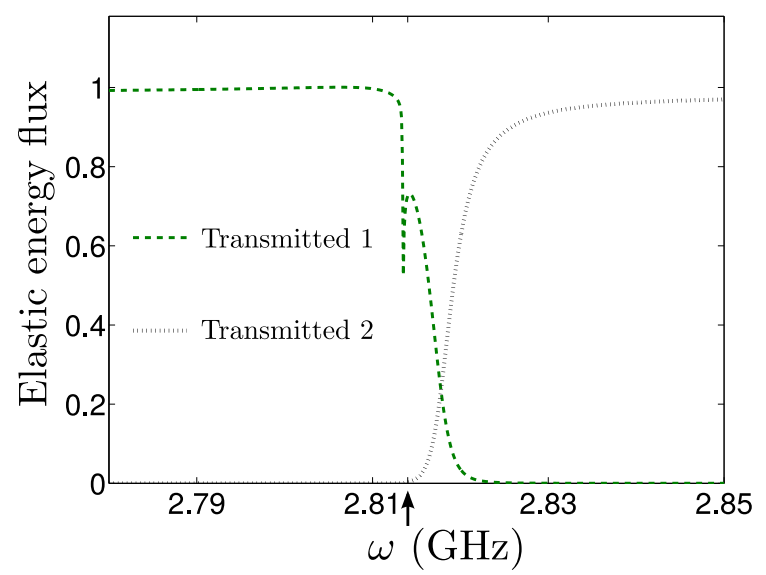

(c)

FIG. 4. (Color online) (a) Normalized energy flux carried by the reflected and transmitted waves. At $\omega_{0}$, wave 2 changes character from evanescent to propagating. (b) Ratio between the elastic and magnetic energy densities associated with the transmitted waves. The ratio becomes very small when the magnetic energy dominates but never vanishes. The shaded region depicts the pseudoband gap for elastic waves. (c) Lattice contribution to the energy flux for the two transmitted waves. The arrows on the abscissas indicate the FMR frequency $\omega_{0}$.

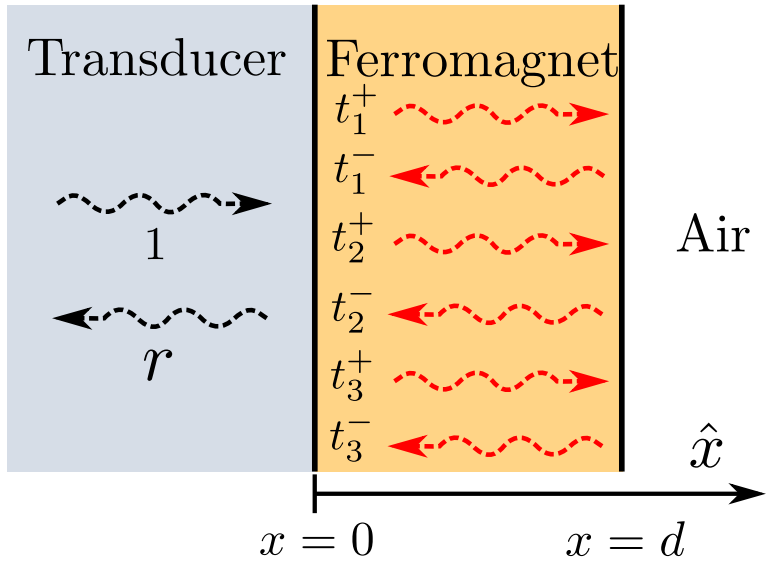

FIG. 5. (Color online) Schematic of an N|F structure exposed to vacuum or air on the $\mathrm{F}$ side. Elastic waves incident from $\mathrm{N}$ excite MEWs in $\mathrm{F}$. The $\mathrm{F} \mid$ vacuum interface at $x=d$ totally reflects all waves. Standing wave solutions in F are broadened by the energy leakage back into $\mathrm{N}$. the boundary conditions [Eqs. (21)-(23)] at $x=0 \mathrm{read}$

$$
\begin{aligned}
\sum_{l}\left(t_{l}^{+} k_{l} \chi_{l}[1]+t_{l}^{-} k_{l} \chi_{l}[1] e^{i k_{l} d}\right) & =0, \\
\sum_{l}\left(t_{l}^{+} k_{l} \chi_{l}[2]+t_{l}^{-} k_{l} \chi_{l}[2] e^{i k_{l} d}\right) & =0,
\end{aligned}
$$

$$
\begin{gathered}
\sum_{l}\left(t_{l}^{+} \chi_{l}[3]+t_{l}^{-} \chi_{l}[3] e^{i k_{l} d}\right)=\sqrt{\frac{2}{\mu_{N} \omega k_{i}}}(1+r), \\
\sum_{l}\left[\left(i \mu_{F} k_{l} \chi_{l}[3]+\frac{b_{2}}{M_{s}} \chi_{l}[1]\right)\left(t_{l}^{+}-t_{l}^{-} e^{i k_{l} d}\right)\right] \\
=i \mu_{N} k_{i} \sqrt{\frac{2}{\mu_{N} \omega k_{i}}}(1-r) .
\end{gathered}
$$

The total reflection corresponds to the free boundary condition at the outer interface $(x=d)$,

$$
\sum_{l} k_{l} \chi_{l}[1]\left(t_{l}^{+} e^{i k_{l} d}+t_{l}^{-}\right)=0,
$$




$$
\begin{gathered}
\sum_{l} k_{l} \chi_{l}[2]\left(t_{l}^{+} e^{i k_{l} d}+t_{l}^{-}\right)=0 \\
\sum_{l}\left[\left(i \mu_{F} k_{l} \chi_{l}[3]+\frac{b_{2}}{M_{s}} \chi_{l}[1]\right)\left(t_{l}^{+} e^{i k_{l} d}-t_{l}^{-}\right)\right]=0
\end{gathered}
$$

thereby completing the set of seven linear equations [Eqs. (33)-(39)] for seven variables $\left(r, t_{l}^{\sigma}\right)$. While no energy is transported it is instructive to plot the normalized energy flux carried by the reflected and the forward traveling waves, corresponding to the coefficients $r, t_{1}^{+}$, and $t_{2}^{+}$, for different $\mathrm{F}$ thicknesses in Fig. 6. The flux of transmitted waves is not bounded by unity now [see Fig. 6(a)]. The sharp feature in the flux of transmitted wave 1 disappears with decreasing $d$, implying that the standing wave excitation in $\mathrm{F}$ is most efficient when the wavelength of the incident elastic wave matches $d$.

The magnetization dynamics or MEW excitation in F can be detected conveniently via spin pumping [23] into a thin ( $\sim$ few $\mathrm{nm}$ ) platinum film [5,7] that converts the spin current into a transverse charge current via the inverse spin Hall effect (ISHE) [24]. The spin current density injected into a thin Pt film [25] contact on F reads [23]

$$
\mathbf{J}^{s}=\left.\frac{g_{r} \hbar}{4 \pi M_{s}^{2}}(\mathbf{M} \times \dot{\mathbf{M}})\right|_{x=d}
$$

where $g_{r}$ is the real part of the spin mixing conductance per unit area [23], and we disregard its imaginary part as well as spin current backflow [26]. The time-averaged spin current is polarized along $\hat{\mathbf{z}}$,

$$
\left\langle\mathbf{J}^{s}\right\rangle_{t}=\left\langle\left.\frac{g_{r} \hbar}{4 \pi M_{s}^{2}}\left(M_{x} \dot{M}_{y}-M_{y} \dot{M}_{x}\right)\right|_{x=d}\right\rangle_{t} \hat{\mathbf{z}}=\frac{g_{r} \hbar \omega}{4 \pi} \beta^{2} \hat{\mathbf{z}},
$$

where we define the "spin pumping angle" [27] $\beta=$ $\sqrt{\operatorname{Im}\left[\mathfrak{m}_{x}^{*} \mathfrak{m}_{y} / M_{s}^{2}\right]}$ as a dimensionless measure of the pumped spin current, with $M_{x, y}(x=d)=\mathfrak{m}_{x, y} e^{-i \omega t}$ and [Eq. (32)]

$$
\mathfrak{m}_{x, y}=\sum_{l} \chi_{l}[1,2]\left(t_{l}^{+} e^{i k_{l} d}-t_{l}^{-}\right) .
$$

The spin current pumped into the Pt film is converted into a transverse voltage by the ISHE that can be computed by solving the spin diffusion equation with the appropriate boundary conditions [28].

The squared spin pumping angle $\beta^{2}$ is proportional to the incident energy flux $F_{\text {in }}$. The ratio $\beta^{2} / F_{\text {in }}$ is plotted against $\omega$ in Fig. 6 (upper panels) for different thicknesses $d$. The spin current is resonantly enhanced around the FMR frequency $\omega_{0}$ with a maximum that decreases with $d$, as expected from the excitation efficiency (lower panels in Fig. 6). A dip in the frequency dependence of the spin pumping angle develops at a frequency slightly below $\omega_{0}$ with decreasing $d$ (upper panels in Fig. 6). This dip is attributed to an enhanced excitation of the evanescent (counter-rotating) $\mathrm{m}^{-}$mode, which pumps spin current with opposite polarity, when $d$ is comparable to or less than the decay length (a few hundred $\mathrm{nm}$ ) of this mode. In configuration 2, the $m^{-}$mode does not couple to the incident $r^{+}$wave, hence the $\beta^{2}$ spectra are almost symmetric Lorentzians (see Fig. 7).

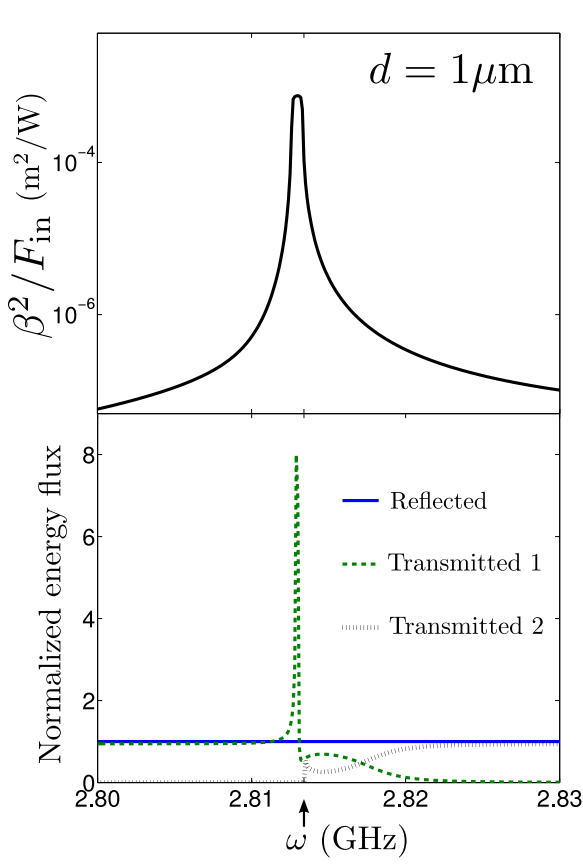

(a)

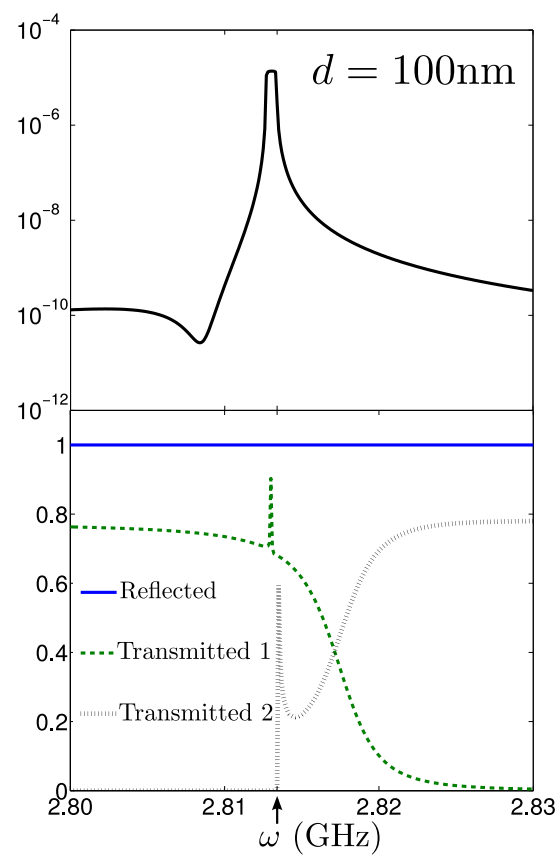

(b)

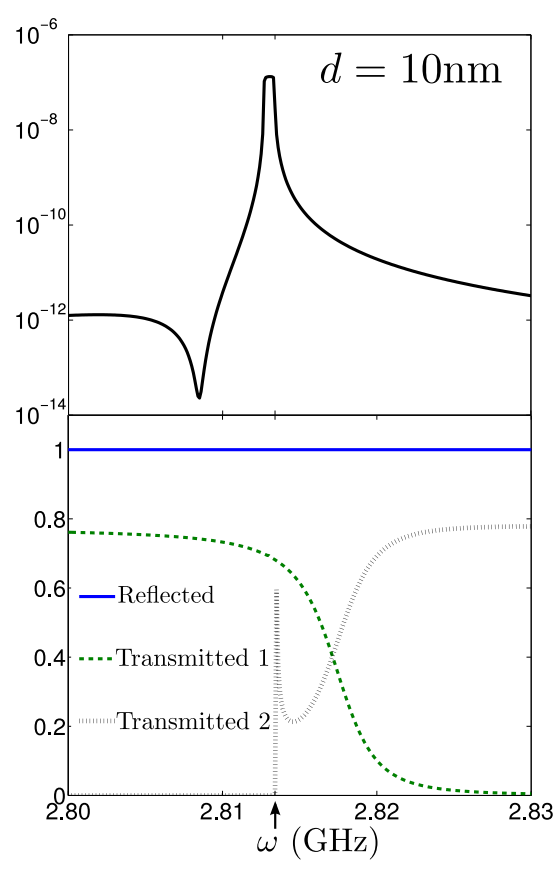

(c)

FIG. 6. (Color online) Energy fluxes and squared spin pumping angle $\beta^{2}$ per incident flux $F_{\text {in }}$ vs frequency $\omega$ of the incident elastic wave for F layer thickness (a) $d=1 \mu \mathrm{m}$, (b) $d=100 \mathrm{~nm}$, and (c) $d=10 \mathrm{~nm}$. Note the different scales on the ordinate. The arrows on the abscissas indicate the FMR frequency $\omega_{0}$. The fluxes shown here are carried by the forward propagating transmitted waves in $\mathrm{F}$ and reflected wave in $\mathrm{N}$. 


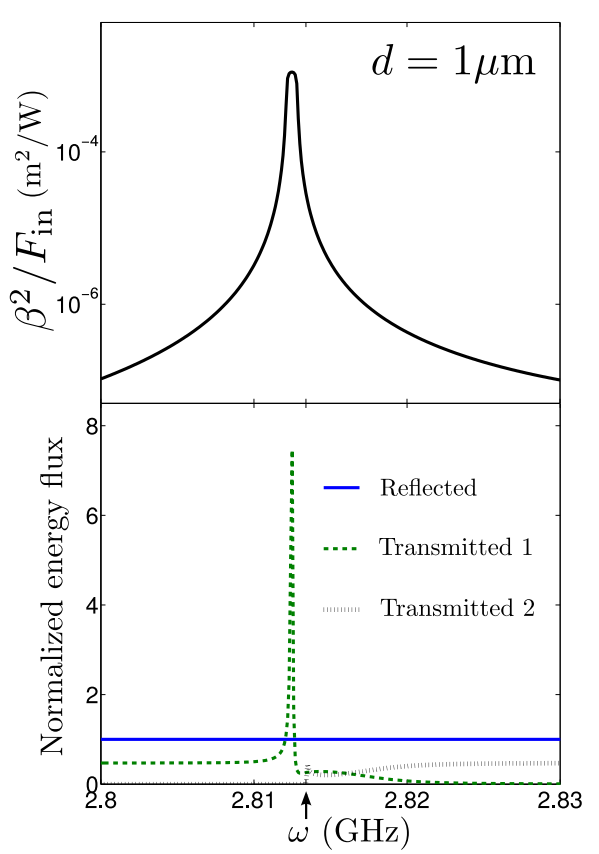

(a)
Config. 2

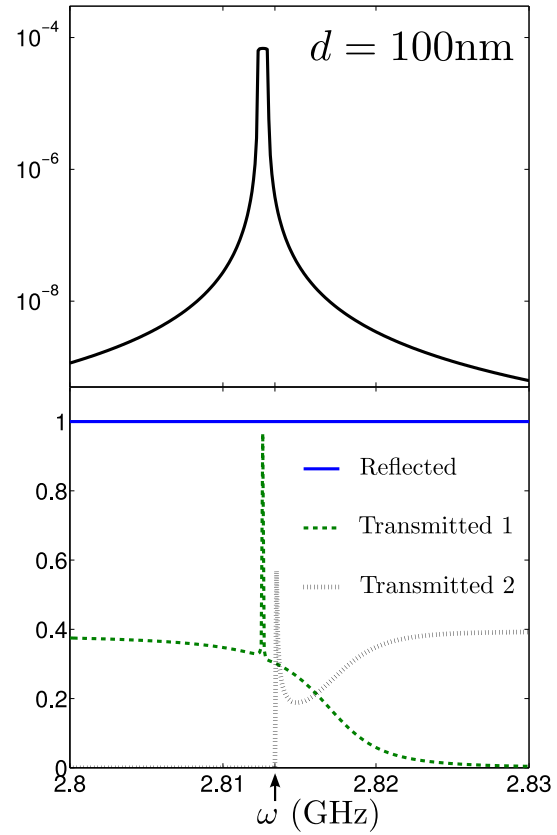

(b)

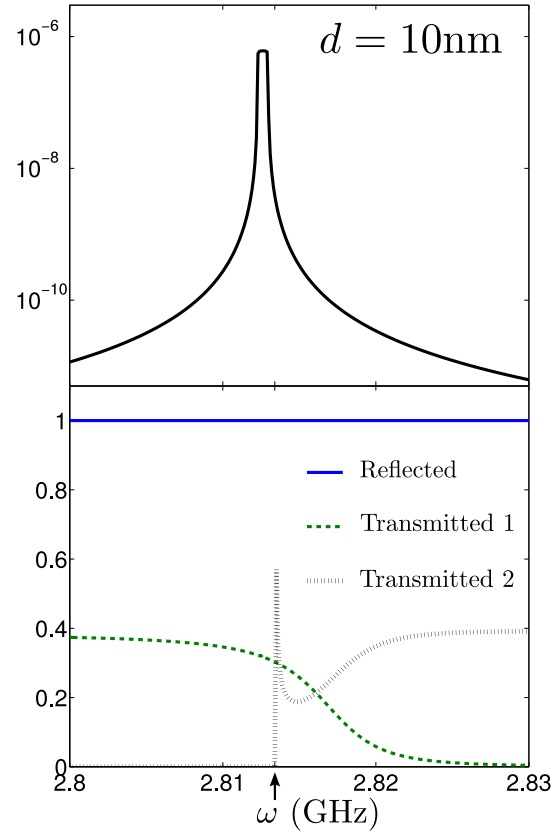

(c)

FIG. 7. (Color online) Configuration 2: Energy fluxes and squared spin pumping angle $\beta^{2}$ per incident flux $F_{\text {in }}$ vs frequency $\omega$ of the incident elastic wave for F layer thickness (a) $d=1 \mu \mathrm{m}$, (b) $d=100 \mathrm{~nm}$, and (c) $d=10 \mathrm{~nm}$. The arrows on the abscissas indicate the FMR frequency $\omega_{0}$. The fluxes shown here are carried by the forward propagating transmitted waves in $\mathrm{F}$ and reflected wave in $\mathrm{N}$.

The maximum value of $\beta^{2} / F_{\text {in }}$ around $\omega_{0}$ as a function of $d$ in Fig. 8(a) shows a peak at $d \approx 0.62 \mu \mathrm{m}$, a thickness comparable to the wavelength of the incident elastic wave. $\beta^{2} / F_{\text {in }}$ is plotted for $d=0.62 \mu \mathrm{m}$ over a wider frequency range in Fig. 8(b). Two additional peaks can be attributed to spin wave resonances $\left(k_{n}=n \pi / d, n=1,2\right)$. A perfect energy sink at the outer interface, as considered in the previous section, suppresses any reflection. The resulting average squared spin pumping angle per incident flux, depicted by the blue dashed line in Fig. 8(b), is indeed considerably smaller than in the case of a reflecting interface.

We note that all the excited modes are dominantly magnonic because the frequencies corresponding to the wave numbers $k_{n}$ lie in the $\mathrm{W}$ region [Fig. 2(b)]. The translational symmetry breaking at the interface allows excitation of spin waves without wave-number conservation. $\left(\beta^{2} / F_{\text {in }}\right)_{\max }$ (and hence the spin current) decreases with increasing $n$.

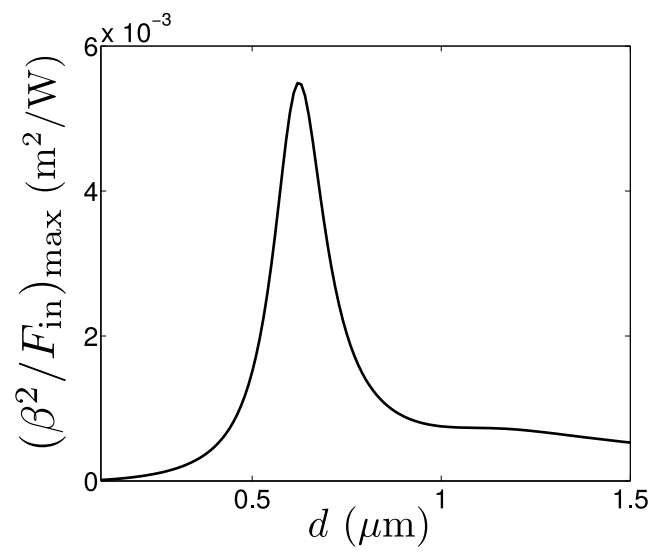

(a)

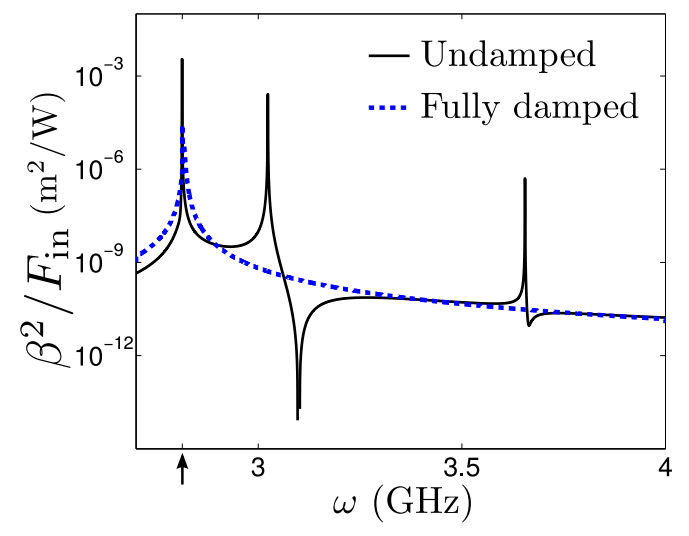

(b)

FIG. 8. (Color online) (a) Maximum value of $\beta^{2} / F_{\text {in }}$ in the frequency range around $\omega_{0}$ vs the thickness of the ferromagnetic film $(d)$. (b) $\beta^{2} / F_{\text {in }}$ vs $\omega$ for $d=0.62 \mu \mathrm{m}$ corresponding to the maximum in (a). The peaks corresponding to the first two standing MEWs, in addition to the uniform mode, can be seen. The fully damped case, corresponding to an ideal acoustic sink at the far end (or an infinitely thick F layer as considered in Sec. III A), is depicted by the dashed line. The arrow on the abscissa indicates the FMR frequency $\omega_{0}$. 


\section{CONCLUSION}

We study the excitation of magnetization dynamics in a ferromagnet $(F)$ via elastic waves injected by an attached nonmagnetic transducer $(\mathrm{N})$. To this end, a scattering theory formulation of the magnetoelastic waves (MEWs) resulting from magnetoelastic coupling (MEC) in F has been employed. We solve the equations of motion for MEWs propagating orthogonal to (configuration 1) and along (configuration 2) the equilibrium magnetization direction. Configuration 1 leads to excitation of evanescent counter-rotating spin waves, in addition to the two traveling quasispin and quasielastic waves. The evanescent waves are not important for energy transport but play significant roles in other phenomena, such as transients in pulsed excitation or evanescent-wave mediated coupling between two media [29].

Acoustic excitation of MEWs can efficiently generate magnetization dynamics in the form of magnon polarons (MPs) around the anticrossing region. In sufficiently thin ferromagnetic films standing spin waves can also be excited. The efficiency is maximized for $F$ layer thicknesses that match the wavelength of the elastic waves. The magnetization dynamics can be detected by spin pumping into an adjacent normal metal layer via the inverse spin Hall effect. The formulation of energy and spin transport by MEWs unifies phononics and magnonics, thereby paving the way into yet unchartered territory.

\section{ACKNOWLEDGMENTS}

A.K. thanks M. Weiler, S. Goennenwein, and H. Huebl for fruitful discussions. This work was supported by the FOM Foundation, Reimei program of the Japan Atomic Energy Agency, EU-FET "InSpin" the ICC-IMR, DFG (Germany) Priority Programme 1538 "Spin-Caloric Transport" (BA2954/2), and JSPS Grants-in-Aid for Scientific Research (Grants No. 25247056, No. 25220910, and No. 26103006).
[1] S. Chikazumi and C. Graham, Physics of Ferromagnetism, International Series of Monographs on Physics (Clarendon, London, 1997).

[2] T. Gilbert, IEEE Trans. Magn. 40, 3443 (2004).

[3] A. Akhiezer, V. Bar'iakhtar, and S. Peletminski, Spin Waves (North-Holland, Amsterdam, 1968).

[4] C. Kittel, Phys. Rev. 110, 836 (1958)

[5] K. Uchida, H. Adachi, T. An, T. Ota, M. Toda, B. Hillebrands, S. Maekawa, and E. Saitoh, Nat. Mater. 10, 737 (2011).

[6] K. Uchida, J. Xiao, H. Adachi, J. Ohe, S. Takahashi, J. Ieda, T. Ota, Y. Kajiwara, H. Umezawa, H. Kawai, G. E. W. Bauer, S. Maekawa, and E. Saitoh, Nat. Mater. 9, 894 (2010).

[7] M. Weiler, H. Huebl, F. S. Goerg, F. D. Czeschka, R. Gross, and S. T. B. Goennenwein, Phys. Rev. Lett. 108, 176601 (2012).

[8] S. Geprägs, A. Brandlmaier, M. Opel, R. Gross, and S. T. B. Goennenwein, Appl. Phys. Lett. 96, 142509 (2010).

[9] A. A. Kovalev, G. E. W. Bauer, and A. Brataas, Phys. Rev. Lett. 94, 167201 (2005).

[10] R. C. LeCraw and R. L. Comstock, in Physical Acoustics, edited by W. P. Mason (Academic, New York, 1965), Vol. IIIB, pp. 127-199.

[11] A. Kamra and G. E. W. Bauer, Solid State Commun. 198, 35 (2014).

[12] S. Datta, Quantum Transport: Atom to Transistor (Cambridge University Press, Cambridge, UK, 2005).

[13] C. Kittel, Rev. Mod. Phys. 21, 541 (1949).

[14] W. Lai, D. Rubin, D. Rubin, and E. Krempl, Introduction to Continuum Mechanics (Elsevier, Amsterdam, 2009).
[15] H. Keshtgar, M. Zareyan, and G. E. W. Bauer, Solid State Commun. 198, 30 (2014).

[16] É. Du Trémolet de Lacheisserie, D. Gignoux, and M. Schlenker, Magnetism (Springer, Berlin, 2005).

[17] R. L. Comstock and B. A. Auld, J. Appl. Phys. 34, 1461 (1963).

[18] For wave propagation along a nonsymmetry direction, longitudinal elastic (pressure) waves also couple to the magnetization dynamics.

[19] F. G. Eggers and W. Strauss, J. Appl. Phys. 34, 1180 (1963).

[20] P. Hansen, Phys. Rev. B 8, 246 (1973).

[21] YIG Crystal Specification Sheet, Deltronic Crystal Industries, Inc .

[22] L. J. Graham and R. Chang, J. Appl. Phys. 41, 2247 (1970).

[23] Y. Tserkovnyak, A. Brataas, and G. E. W. Bauer, Phys. Rev. Lett. 88, 117601 (2002).

[24] E. Saitoh, M. Ueda, H. Miyajima, and G. Tatara, Appl. Phys. Lett. 88, 182509 (2006).

[25] The Pt film does not affect the boundary condition considerations when it is much thinner than the inverse of the wave number that amounts to a few $\mu \mathrm{m}$ in the $\mathrm{GHz}$ regime.

[26] H. J. Jiao and G. E. W. Bauer, Phys. Rev. Lett. 110, 217602 (2013).

[27] $\beta$ is approximately equal to the precession cone angle.

[28] O. Mosendz, V. Vlaminck, J. E. Pearson, F. Y. Fradin, G. E. W. Bauer, S. D. Bader, and A. Hoffmann, Phys. Rev. B 82, 214403 (2010).

[29] K. Uchida, H. Adachi, D. Kikuchi, S. Ito, Z. Qiu, S. Maekawa, and E. Saitoh, Nat. Commun. 6, 5910 (2015). 